\title{
Arrangement of Ceramides in the Skin: Sphingosine Chains Localize at a Single Position in Stratum Corneum Lipid Matrix Models
}

\author{
Charlotte M. Beddoes, Gert S. Gooris, Fabrizia Foglia, Delaram Ahmadi, David J. Barlow, \\ M. Jayne Lawrence, Bruno Demé, and Joke A. Bouwstra*
}

Cite This: Langmuir 2020, 36, 10270-10278

Read Online

ACCESS

Wll Metrics \& More

Article Recommendations

Supporting Information

ABSTRACT: Understanding the structure of the stratum corneum (SC) is essential to understand the skin barrier process. The long periodicity phase (LPP) is a unique trilayer lamellar structure located in the SC. Adjustments in the composition of the lipid matrix, as in many skin abnormalities, can have severe effects on the lipid organization and barrier function. Although the location of individual lipid subclasses has been identified, the lipid conformation at these locations remains uncertain. Contrast variation experiments via smallangle neutron diffraction were used to investigate the conformation of ceramide (CER) N(tetracosanoyl)-sphingosine (NS) within both simplistic and porcine mimicking LPP models.

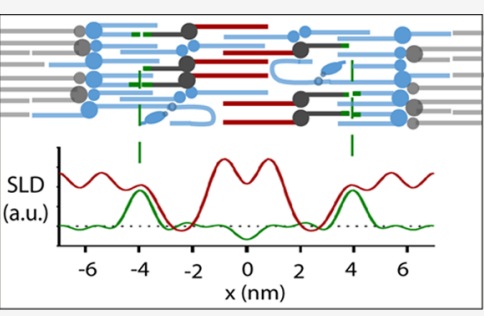
To identify the lipid conformation of the twin chain CER NS, the chains were individually deuterated, and their scattering length profiles were calculated to identify their locations in the LPP unit cell. In the repeating trilayer unit of the LPP, the acyl chain of CER NS was located in the central and outer layers, while the sphingosine chain was located exclusively in the middle of the outer layers. Thus, for the CER NS with the acyl chain in the central layer, this demonstrates an extended conformation. Electron density distribution profiles identified that the lipid structure remains consistent regardless of the lipid's lateral packing phase, this may be partially due to the anchoring of the extended CER NS. The presented results provide a more detailed insight on the internal arrangement of the LPP lipids and how they are expected to be arranged in healthy skin.

\section{INTRODUCTION}

Lipids are an essential component for the bodies signaling network, ${ }^{1,2}$ energy storage, ${ }^{3}$ and cellular membranes. Sphingolipids, particularly ceramides (CERs), are critical for effective barrier control of the skin. The precursors of the lipids are glucosylceramides and sphingomyelin. Together with phospholipids and cholesterol (CHOL), these lipids form the main components of the viable membranes in the skin. The skin is one of the major defenses the body has against the penetration of materials from the external environment and desiccation. The upper layer of the skin, the stratum corneum (SC), is the main barrier that these materials must permeate through and thus determines the skin barrier's effectiveness. ${ }^{4}$ The SC consists of corneocytes embedded in a lipid matrix; it is this matrix that forms the only continuous structure through the SC, thus it is considered to have a critical role in the barrier's function. ${ }^{5,6}$ Aside from the CERs, the main lipid classes in the SC include CHOL and free fatty acids (FFAs), present in an approximately equimolar ratio. These lipids form two crystalline lamellar phases with repeat distances of approximately 6 and $13 \mathrm{~nm} .^{7,8}$ The $13 \mathrm{~nm}$ lamellar phase is referred to as the long periodicity phase (LPP) and is unique to the SC. Currently, 18 CER subclasses have been identified from human SC, ${ }^{9-11}$ which are typically referred to by their nomenclatures based on the definitions from Motta et al. ${ }^{12}$ The correct lipid arrangement within these lamellar structures is essential for skin barrier function, as many inflammatory skin diseases are often related to identifiable changes in the lipid matrix composition and arrangement. ${ }^{10,13,14}$

Mixtures based on either isolated CERs or synthetic CERs showed that these lipid mixtures can closely resemble the lipid organization and permeability in human SC. ${ }^{15-20}$ Using these lipid matrix models (LMMs), the location of the major lipid subclasses within the LPP unit cell has been previously identified with the use of contrast variation small-angle neutron diffraction (SAND) measurements, including CERs $\mathrm{N}$ (tetracosanoyl)-sphingosine (NS) with an acyl chain of C24 (CER NS (C24)), CER N-(30-linoleoyloxy-triacontanoyl)sphingosine (EOS) with an acyl chain of C30 (CER EOS (C30)), FFAs and CHOL. ${ }^{21-23}$ However, information on how the CERs are arranged at their locations, such as conformational order, remains incomplete.

Twin chained lipids, such as CERs, are able to arrange themselves into either a hairpin or an extended conformation. When both chains are located on the same side of the headgroup, the conformation is referred to as a hairpin, while if the two tails are located on either side of the headgroup, then

Received: July 7, 2020

Revised: August 11, 2020

Published: August 20, 2020 
the lipid is arranged in an extended conformation. Currently, in the arrangement for the trilayer structure of the LPP reported by Mojumdar et al., ${ }^{22}$ infrared spectroscopy measurements indicate that CER NS is extended within the trilayer structure. $^{24}$ However, stronger evidence of the extended structure is still needed. Knowledge of whether the CERs are able to protrude into neighboring lamellar layers, both internally and externally from the unit cell, would provide more insight into the molecular mechanism underlying the barrier function of the LPP structure.

In this study, we have investigated the arrangement and conformation of CER NS within the LPP unit cell using synthetic models that form the LPP structure exclusively. Two models were compared, one that closely resembles the lipid composition of porcine SC (the porcine model), while maintaining similar phase behavior of human SC, as well as a model that contains the fewest number of CER subclasses as possible to form the LPP (the simple model). The porcine SC model was selected, due to its similar phase behavior as found in human SC and in isolated CER models, ${ }^{15,25}$ and of which most CER subclasses are available. The hydrogen linked to the terminal three carbons of the sphingosine chain of CER NS as well as the full acyl chain of CER NS was deuterated to identify the location of each of these moieties, together with the overall conformation of this CER subclass. We also show how the CER arrangement assists in maintaining the LPP structure by measuring the internal structural rearrangement during the lipid's lateral phase transitions.

\section{EXPERIMENTAL SECTION}

Materials. CER EOS (C30) as well as shorter CERs, including CER NS (C24), N-(tetracosanoyl)-phytosphingosine (CER NP (C24 and C16)), N-(2R-hydroxy-tetracosanoyl)-sphingosine (CER AS (C24)), and $\mathrm{N}$-(2R-hydroxy-tetracosanoyl)-phytosphingosine (CER AP (C24)), was all kindly donated by Evonik, Essen, Germany. Palmitic acid (C16), stearic acid (C18), arachidic acid (C20), behenic acid (C22), tricosylic acid (C23), lignoceric acid (C24), cerotic acid (C26), and CHOL were purchased from Sigma-Aldrich Chemie $\mathrm{GmbH}$, Schnelldorf, Germany. The structures of both partially deuterated CER NS (C24), which are substituted into the models, are shown in Figure 1. These included: when hydrogens on the terminal three carbons of the sphingosine chain were replaced with deuterium (CER NS-d7, purchased from Avanti Polar Lipids, Alabama) and when the hydrogen atoms along the entire acyl chain were also replaced (CER NS-d47, kindly provided by Evonik, Essen, Germany). All solvents used were of analytical grade and supplied by Labscan, Dublin, Ireland. The water was of Millipore quality produced by a

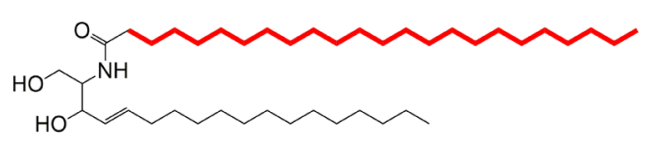

CER NS-d47

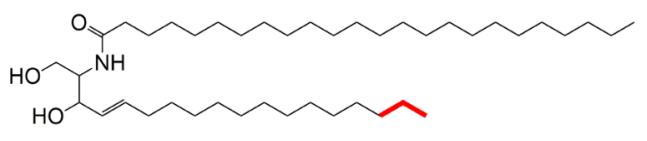

CER NS-d7

Figure 1. Molecular structure of deuterated CERs used in this study. This includes CER NS-d47 where all 47 hydrogen atoms along the acyl chain were replaced with deuterium and CER NS-d7 where the terminal seven hydrogen atoms of the sphingosine chain were replaced with deuterium. The carbon atoms bound to deuterium are highlighted in bold.
Milli-Q water filtration system with a resistivity of $18 \mathrm{M} \Omega \mathrm{cm}$ at 25 ${ }^{\circ} \mathrm{C}$. Nucleopore polycarbonate filters, with $0.05 \mu \mathrm{m}$ pore size, were purchased from Whatman, Kent, U.K.

Model Composition. Two different models were investigated comprising of either a two CER subclass model (CER EOS and NS, the simple model) or a porcine SC mimicking model (porcine model). Each model was prepared from synthetic CERs, CHOL, and FFAs in an equimolar ratio to mimic the composition of SC. The ratios of the specific lipid subclasses are presented in Table 1.

Table 1. Lipid Components and Molar Ratio for the Simple and Porcine Models

\begin{tabular}{lcc}
\multicolumn{1}{c}{ lipid subclass } & $\begin{array}{c}\text { simple model } \\
(\text { molar \% })\end{array}$ & $\begin{array}{c}\text { porcine model } \\
(\text { molar \% })\end{array}$ \\
CER EOS (C30) & 13.3 & 13.33 \\
CER NS/NS-d7/NS-d47 (C24) & 20 & 12 \\
CER NP (C24) & & 3.7 \\
CER AS (C24) & & 1 \\
CER NP (C16) & & 2 \\
CER AP (C24) & 33.3 & 1.3 \\
cholesterol & 0.6 & 33.3 \\
palmitic acid (C16) & 1.33 & 0.6 \\
stearic acid (C18) & 2.53 & 1.33 \\
arachidic acid (C20) & 15.93 & 2.57 \\
behenic acid (C22) & & 14.2 \\
tricosylic acid (C23) & 12.93 & 1.73 \\
lignoceric acid (C24) & & 11.57 \\
cerotic acid (C26) & & 1.37
\end{tabular}

Regardless of the model used, CER EOS was fixed to $13.3 \mathrm{~mol} \%$ of the total lipid content, to ensure that the LPP would form exclusively. ${ }^{26,27}$ For the partially deuterated measurements, CER NS was substituted with either CER NS-d7 or CER NS-d47.

Lipid Model Preparation. For all SAND measurements, $10 \mathrm{mg}$ of lipids, in the appropriate molar proportions, was dissolved in chloroform/methanol $(2: 1 \mathrm{v} / \mathrm{v})$ to a concentration of $5 \mathrm{mg} / \mathrm{mL}$. Over an area of $40 \times 13 \mathrm{~mm}^{2}$, the samples were sprayed on a silicon wafer using a y-axis adapted Camag Linomat IV sample applicator (Muttenz, Switzerland) under a steady stream of nitrogen. Once sprayed, the samples were heated until melted $\left(65-70{ }^{\circ} \mathrm{C}\right)$ and cooled back to room temperature and heated again for a total of two cycles, ultimately the samples were melted for a total of $30 \mathrm{~min}$. Once equilibrated, the samples were then hydrated in $\mathrm{D}_{2} \mathrm{O} / \mathrm{H}_{2} \mathrm{O}$ mixtures at ratios of 100:0, 50:50, and $8: 92(\mathrm{v} / \mathrm{v})$ in $100 \%$ relative humidity, initially for $\sim 18 \mathrm{~h}$, at $32{ }^{\circ} \mathrm{C}$. Once measured, the samples were hydrated to the next water ratio for at least $8 \mathrm{~h}$ at $32{ }^{\circ} \mathrm{C}$, to ensure complete solvent exchange in the LPP. A complete list of sample compositions can be found in the Supporting Information (Table S1).

The small-angle X-ray diffraction (SAXD) samples were prepared using a similar method; however, $0.90 \mathrm{mg}$ of lipids was dissolved in hexane/ethanol (2:1) and sprayed over an area of $10 \times 10 \mathrm{~mm}^{2}$ on the polycarbonate filters and equilibrated at $85{ }^{\circ} \mathrm{C}$ for $30 \mathrm{~min}$ for a single cycle. A single, higher equilibration temperature was possible for the SAXD samples due to the lipid's greater adhesive strength with these filters. The samples were hydrated in a $100 \% \mathrm{H}_{2} \mathrm{O}$ humid environment for at least $\sim 18 \mathrm{~h}$ before measuring.

D16 Neutron Diffractometer Measurements. Neutron diffraction experiments were performed on the D16 neutron diffractometer at the Institut Laue-Langevin, Grenoble, France. The measurement and data analysis procedure have been described previously. ${ }^{21,22}$ In brief, the incoming slit-collimated beam (wavelength $4.52 \AA$ ) was set to $25 \mathrm{~mm}$ vertically and $4 \mathrm{~mm}$ horizontally, to ensure that the entire sample remains in the beam for all diffraction order measurements. The diffraction patterns were measured in reflection mode, with the sample positioned $0.950 \mathrm{~m}$ from the $320 \times$ $320 \mathrm{~mm}{ }^{3} \mathrm{He}$ detector (which provided a spatial resolution of $1 \times 1$ $\mathrm{mm}$ ). The samples were mounted in an aluminum humidity 
chamber, ${ }^{28}$ maintained at $25{ }^{\circ} \mathrm{C}$ and measured for a total of $2-6 \mathrm{~h}$ depending on the signal to noise ratio. The samples were rotated between 0.05 and $10.2^{\circ}$ and measured in $0.05^{\circ}$ steps to cover the first 9 diffraction orders. For each diffraction order, the scan measured at the specular angle and $\pm 0.1^{\circ}$ (culminating to a total of 5 scans), an example of which is shown in the Supporting Information (Figure S1), were averaged together and fitted. The scattering data were reduced, background-subtracted, and the peaks were fitted using the data processing software LAMP. ${ }^{29}$ While converting $2 \theta$ into to $\mathrm{q}$ spacing, a rearranged Bragg equation was used

$$
q=\frac{4 \pi \sin \theta}{\lambda}
$$

When in the lamellar phase, a series of peaks at equal q-distances to one another are detected. The repeat distance $(d)$ of the lamellar phase can be calculated from the positions of the peaks as

$$
d=\frac{2 n \pi}{q_{n}}
$$

with $n$ as the order number of the diffraction peak located at position $q_{n}$.

Scattering Length Density (SLD) Calculations. Scattering curves were analyzed with a similar method that has been reported previously. ${ }^{20,21}$ In short, all diffraction orders were fitted with a Gaussian function to determine the scattering intensity $(I)$. The $I$ of the peaks were used to calculate the structure factor amplitude $\left(\left|F_{n}\right|\right)$ for that order. The $\left|F_{n}\right|$ was calculated by

$$
\left|F_{n}\right|=A_{n} \sqrt{L I}
$$

where $L$ is the Lorentz correction, due to the high degree of orientation in the sample; it can be calculated as $L=n$. $A_{n}$ is the correction factor for sample absorption, which is calculated as the following ${ }^{30}$

$$
A_{n}=\frac{1}{\sqrt{\frac{\sin \theta}{2 \mu l}}\left(1-\mathrm{e}^{-2 \mu l / \sin \theta}\right)}
$$

where $\mu$ refers to the linear attenuation coefficient and $l$ is the lipid thickness.

The issue with the scattering experiments is that the information on the phase sign is lost. The LPP has been identified to be centrosymmetric. This has been illustrated by the linear fitting of the structure factor $\left(F_{n}\right)$ values as a function of the $\mathrm{D}_{2} \mathrm{O} / \mathrm{H}_{2} \mathrm{O}$ ratio ${ }^{22}$ (Supporting Information, Figure S2). First we determined the scattering phase signs; in these models, the scattering length density (SLD) phase signs for the first 9 diffraction orders of the LPP were assigned as,,,,,,,,-+-+-+-+- , this was the only combination that located the water molecules at the expected unit cell border, due to the hydrophilic interactions of the lipid headgroups. This combination also resulted in a second maximum at approximately 2 $\mathrm{nm}$ from the center of the unit cell, while other phase sign combinations resulted in unrealistic water profiles. These phase signs coincidentally match with previously reported phase signs for water in the LPP. ${ }^{20-22}$ Then, the phase sign order of the LPP for the remaining protiated and each of the deuterated samples were also individually determined and were found to have the same phase orders of,,,,,,,,-+-+-+-+- .

Once the $F_{n}$ was determined, the SLD profile across the unit cell $(\rho(x))$ was calculated by Fourier reconstruction.

$$
\rho(x)=F_{0}+2 \sum_{n=1}^{n_{\max }}\left(F_{n} \cos \frac{2 \pi n x}{d}\right)
$$

where $x$ is the direction of the unit cell and $x=0$ being the center of the unit cell. The zero structure factor order $\left(F_{0}\right)$ is equal to the scattering density per unit volume, ${ }^{31}$ the calculated values for each model are presented in the Supporting Information (Method S1). The "relative absolute" scale was then calculated by determining the scaling factor from the difference in the scattering area between the protiated and deuterated profiles. ${ }^{22,32,33}$ A description on converting to a relative absolute scale can be found in the Supporting Information (Method S1).

Small-Angle X-ray Diffraction Measurements. Small-angle Xray diffraction (SAXD) measurements were performed at the European synchrotron radiation facility (ESRF, Grenoble) at station BM26. The wavelength was set at $1.033 \AA$, and the detector distance was fixed at $2.16 \mathrm{~m}$. A Pilatus $1 \mathrm{M}$ detector was used, the sensor was a reverse-biased Si diode array, consisting of an array of $981 \times 1043$ pixels of a size of $172 \times 172 \mu \mathrm{m}^{2}$. The calibration was performed using silver behenate. The simple model was measured for $60 \mathrm{~s}$ at 1 ${ }^{\circ} \mathrm{C}$ at $25,39,61$, and $67{ }^{\circ} \mathrm{C}$. The one-dimensional (1D) intensity profiles were determined by integrating the two-dimensional (2D) pattern over a segment of $40^{\circ}$ perpendicular to the orientation of the sample, of which the center point was located at the beam center.

Electron Density Distribution (EDD) Calculations. The electron density distribution (EDD) profiles were calculated similarly to the SLD profiles as described earlier. However, the SAXD orders were fitted with a Lorentzian function, which provided the best peak fit, to determine $I$. $\left|F_{n}\right|$ was calculated using eq 3 . However, the X-ray samples scattered similarly to a nonorientated material; thus, when calculating the Lorentz factor for X-ray samples, $L=n^{2}$. Secondary, $A_{n}$ is negligible. ${ }^{20,34}$ This is due to the perpendicular alignment of the $\mathrm{X}$ ray samples to the beam during the measurement, resulting in the smallest possible scattering angle distance, thus the correction for $A_{n}$ is negligible in this instant.

Since the EDD profiles compare the samples at different temperatures, the overall scattering intensity is expected to change. As a result, each peak intensity was normalized in relation to the scan's overall peak intensity. The EDD phase signs were identified using the method previously reported. ${ }^{20}$ As our models do not swell upon hydration, in the previous study, a continuous Fourier was determined from mixtures forming a similar LPP with small variations in repeat distance. When taking the same phase signs, our $F_{n}$ values fitted to the continuous Fourier, indicating that the LPP in this measurement had the same phase signs with the previous study. EDD profiles at a given position in the unit cell were calculated using eq 5 .

Modeling the LPP Neutron Diffraction Distribution. Due to the large error that is inherent with the SLD and EDD profiles, it is important to distinguish true SLD distributions from that caused by data truncation error. Using the lipid arrangement in the LPP of the simple model, the SLD profile was calculated from the identified scattering regions in the LPP unit cell and compared with the experimentally derived SLD profile. The scattering length density was created as follows: $\mathrm{CH}_{2}$ groups have a neutron scattering length of almost $0(-0.83 \mathrm{fm})$, thus aside from the acyl chains of the lipids, all other groups would have a scattering length density value. Using this information, we were able to approximate the scattering length density value for that position of the LPP, this includes contributions from the lipid headgroup/water region located at the LPP boundary and at the inner water layers and the location of the ester group of CER EOS near the inner water layer. Using this theoretical SLD profile from the models, the respective $F_{n}$ values for every order were calculated based on the description by Franks ${ }^{35}$

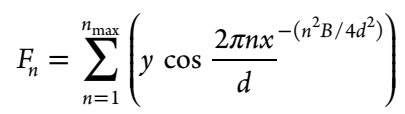

where $y$ is the scattering length density value at position $x$ and $B$ is similar to the Debye-Waller temperature factor to account for repeat lipid disorder. Using the calculated $F_{n}$, the SLD profiles were calculated using the first nine orders and eq 6 and compared with their experimentally derived values. Using these calculated values, they were then adjusted until the resulting SLD profile matched the true SLD profile that was derived from the experimental data.

\section{RESULTS AND DISCUSSION}

Position of CER NS Using Scattering Length Density Profiles. The location of individual lipid subclasses within SC 

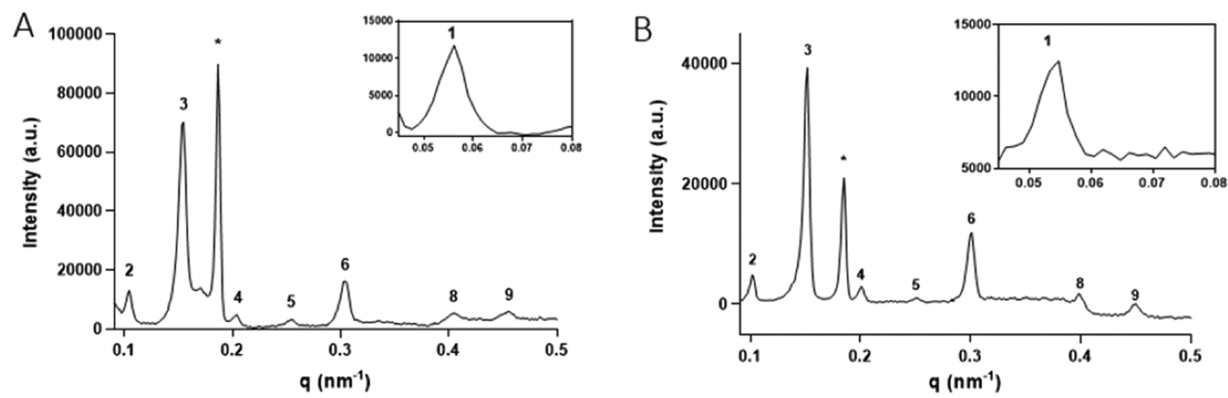

Figure 2. Small-angle neutron $1 \mathrm{D}$ scattering plots of the fully protiated (A) simple and (B) porcine models when hydrated in $50: 50 \mathrm{D}_{2} \mathrm{O} / \mathrm{H}_{2} \mathrm{O}$, measured at a detector position of $13^{\circ}$. All plots were hydrated using $50: 50 \mathrm{D}_{2} \mathrm{O} / \mathrm{H}_{2} \mathrm{O}$. The Arabic numbers indicate the various diffraction orders of the LPP; the 7th order diffraction peak is not visible in both spectra, indicating that the scattering intensity of this order is close to zero. The * indicates the position of the crystalline cholesterol peaks. The insert reports the 1st order diffraction peak measured at a detector position of $11.2^{\circ}$.
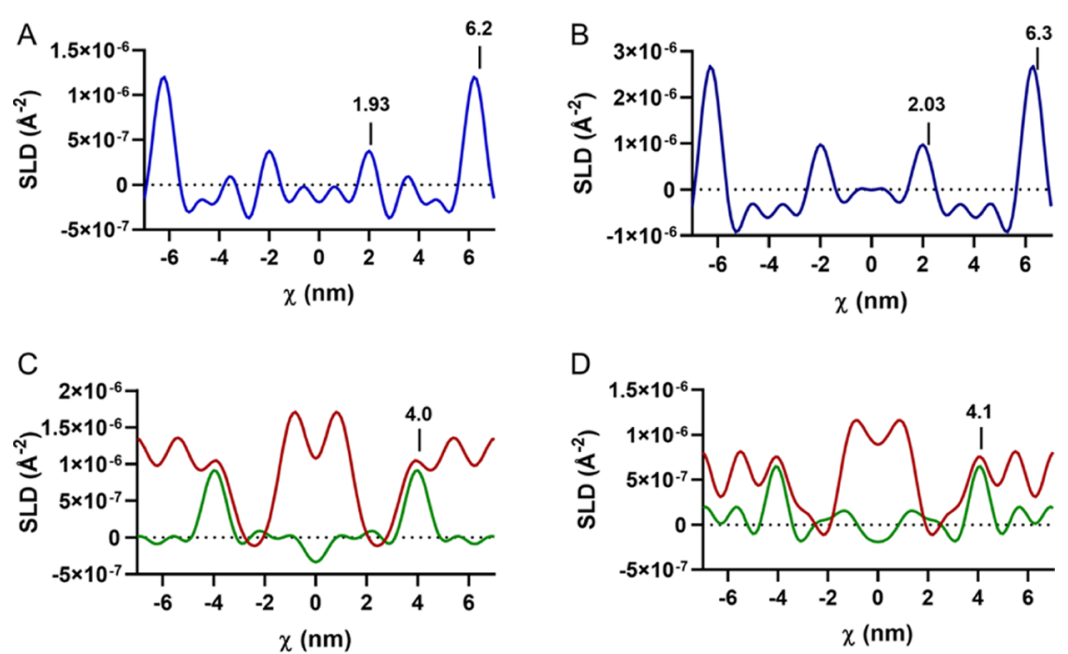

Figure 3. Water SLD profiles of the (A) simple and (B) porcine models within the LPP. Four areas of greater scattering length density signify a trilayer structure. The position of the CER NS chains in the (C) simple and (D) porcine models including the sphingosine $\mathrm{d} 7$ (green) and the acyl chain $\mathrm{d} 47$ (red) profiles. CER NS acyl chains are located in both the inner and outer layers of the LPP, while the sphingosine chain extends beyond the inner water region, locating itself in the outer layers.

lipid structures has been previously studied with the use of neutron diffraction. ${ }^{22,23,36,37}$ However, the specific conformation of CERs has yet to be fully understood. Due to the twin carbon chain structure, CERs are able to adopt either a hairpin or extended conformation. To identify the conformation, the position of both the sphingosine and the acyl chain of CER NS, within the LPP unit cell, was determined with the use of SAND. The 1D diffraction profiles identified up to the $9^{\text {th }}$ diffraction order for all samples. Example curves for the fully protiated simple and porcine models are presented in Figure 2. All sample repeat distances were calculated from least square fitting of the lamellar peaks measured at the detector angle of $13^{\circ}$, which includes all diffraction orders of $\geq 3$. A peak asymmetry is observed in the diffraction curves due to the large sample area, when compared to the detector distance. This becomes more prevalent as the detector angle is reduced, hence the ability to correctly fit the peaks decreases, and thus the first two Bragg peaks measured at the detector angle of $11.2^{\circ}$ are not included in the calculation of the LPP $\mathrm{d}$-spacing. The average repeat distance for the simple model is $12.46 \pm$ $0.08 \mathrm{~nm}$, while the porcine model is $12.57 \pm 0.19 \mathrm{~nm}$. Aside from the Bragg peaks attributed to the LPP, there are also peaks arising from crystalline CHOL (formed due to excess cholesterol that is not incorporated into the LPP) that are observed at $q=0.185$ and $0.368 \AA^{-1}$, equating to a d-spacing of
3.4 and $1.7 \mathrm{~nm}$, respectively. The peaks attributed to the crystalline cholesterol do not overlap with the LPP reflections in any of the samples. No additional peaks that could indicate additional phases were found in the models.

In this study, we selected two variations (simple and porcine) of the LPP SC model. The simple model contains only a few different lipids and can provide unambiguous information on the interactions between specific lipid subclasses. ${ }^{24,27,37,38}$ The porcine model contained a wider variation of CERs and FFAs that closely mimic the composition found in porcine SC. ${ }^{20}$ Models that mimic the composition of human $\mathrm{SC}^{38,39}$ are being developed, but when comparing human and porcine like LMMs, currently porcine models are easier to work with since most of the CER subclasses are readily available, and the lamellar organization closely mimics the lamellar organization in human SC even at elevated temperatures. ${ }^{40}$ In comparison, some CER subclasses present in human SC at relatively higher concentrations remain commercially unavailable.

A major difference the models have compared with porcine SC is the concentration of CER EOS. Native skin has a CER EO content of $\sim 12 \mathrm{~mol} \%$ of the total CER content, ${ }^{9,10}$ which enables the lipids to form both the LPP and SPP. As the aim of the present study was to determine the conformation of CER NS in the LPP, it was important that this was the only 
structure formed. An increase in the CER EOS has been previously shown to increase the proportion of LPP in the model, and by increasing the total CER EOS content to $40 \mathrm{~mol}$ $\%$ of the total CERs, the SPP is lost, leaving only the LPP without affecting the structure and does not result in Bragg peaks to overlap with the CHOL peaks and thus can be a suitable substitution for LPP only studies. ${ }^{26}$

The water profiles were determined by the difference in the SLD profile values when hydrated in the 100:0 $\mathrm{D}_{2} \mathrm{O}$ and the 8:92 $\mathrm{D}_{2} \mathrm{O} / \mathrm{H}_{2} \mathrm{O}$ solvents. The SLD profile over the length of the LPP unit cell (Figure 3) identifies that both the simple and porcine lipid composition models have two water regions, located at the exterior of the unit cell and closer to the center of the centrosymmetric cell, indicating a trilayer structure. The positions of the water peaks were calculated as the averaged position found in the fully protiated $\mathrm{d} 47$ and $\mathrm{d} 7$ versions of each model. The outer water region in the simple and porcine models is located at 6.2 and $6.3 \mathrm{~nm}$, whereas a second water region is present at 1.9 and $2.0 \mathrm{~nm}$ from the unit cell center, where the standard deviations of these positions were 0.2 and $0.1 \mathrm{~nm}$, respectively. This results in the outer layer length slightly exceeding that of the inner layer of the LPP (Table 2). The individual layer length ratios of the LPP structure reported with both models match well with the previously reported results. $^{21}$

Table 2. Length Percentage Ratios between the Outer and Inner Layers of the LPP

\begin{tabular}{lcc} 
sample & outer layer length (\%) & inner layer length (\%) \\
simple & 34.2 & 31.5 \\
porcine & 33.9 & 32.1 \\
\hline
\end{tabular}

The location of the deuterated moieties was identified by the subtraction of the fully protiated SLD profile from their deuterated lipid containing counterpart, when hydrated in the 8:92 $\mathrm{D}_{2} \mathrm{O} / \mathrm{H}_{2} \mathrm{O}$ solvent. The difference in the SLD profiles identified that SLD intensity and thus the location of the C24 acyl chains of the CER NS (Figure 3C,D, red curves) were present in the inner layer and in the outer layers, extending $\sim 2.2 \mathrm{~nm}$ from the exterior of the unit cell. When identifying the location of CER NS's sphingosine chain, the hydrogen atoms of the terminal three carbons were deuterated. The SLD profiles (Figure 3C; green curve) identified that the deuterated groups were located only at 4.0 and $4.1 \mathrm{~nm}$ from the center, $\sim 2 \mathrm{~nm}$ from the inner water/headgroup region in both models. Implying that the sphingosine is located exclusively at this position in the unit cell. From these results, we can conclude that the CER NS located in the center of the unit is entirely arranged in an extended conformation with the acyl chain in the inner layer and the sphingosine chain located in the adjacent layer. When in the outer layer, the location of the CER acyl chains is limited to near the exterior lipid headgroups, but since the terminal chain of the sphingosine is located at the center of the outer layers, we are unable to determine if this sphingosine chain is located in the same layer thus forming an hairpin or located in the neighboring unit cell as an extended conformation.

Our observations extend the LPP molecular arrangements determined by Mojumdar et al. ${ }^{22}$ An extended conformation of CER NS with an acyl chain of C24 has additionally been suggested in the LPP, ${ }^{2,24}$ shorter bilayer structures ${ }^{41,42}$ and in simulated studies. ${ }^{43,44}$ When in its precursor state as a glucosylceramide, the lipid is in a hairpin arrangement. ${ }^{45}$ However, once hydrolyzed into a ceramide, both the size of the CER headgroup and the amount of bound water greatly reduce. The reduction in steric hindrance significantly reduces the conformational exchange between the hairpin and extended structure half time, which would enable CER conformational rearrangement from the initial hairpin, to an extended arrangement. ${ }^{46}$ This suggests that this CER rearrangement is energetically feasible under physiological conditions. The extended conformation of CERs between the inner and outer layers of the LPP, simultaneously with CER EOS, affords a connection between the adjacent lipid layers and so reduces permeability ${ }^{47}$ and discourages swelling within the LPP upon hydration. ${ }^{48}$ An extended configuration also reduces the polar headgroup cross section, and this provides for a higher lipid packing density and reduces packing strain. ${ }^{49,50}$

From the SLD profile, we can also identify the linearity of the CER chains. Assuming that a typical $\mathrm{C}-\mathrm{C}$ bond has a length of $0.15 \mathrm{~nm}$ and when viewed in projection on the major axis, the observed length is close to $0.125 \mathrm{~nm}$. When in an extended conformation, a total of $15 \mathrm{C}-\mathrm{C}$ bonds from the sphingosine chain contributes to a measurable length of 18.75 $\mathrm{nm}$ (Supporting Information, Figure S3). The SLD profile identifies that the terminal of the chain is also located at this position, implying that the sphingosine chain is arranged linearly, perpendicular to the headgroup region.

The acyl chain of CER NS consists of $23 \mathrm{C}-\mathrm{C}$ bonds, which equates to a projected length of 2.875 or $5.77 \mathrm{~nm}$ when mirrored in a bilayer. In contrast, the length of the inner layer is $3.92 \mathrm{~nm}$ in the simple model, $4.03 \mathrm{~nm}$ in the porcine model, and $3.77 \mathrm{~nm}$ in the EDD calculations, are shorter than the bilayer length of the acyl chains, implying that the chains must either be tilted or interdigitated to occupy the finite space.

The average LPP inner layer length in the models is $3.9 \pm$ $0.1 \mathrm{~nm}$; however, the C24 CER chains have a length of 2.88 $\mathrm{nm}$ (Supporting Information, Figure S3); thus, if the chains were tilting, to pack terminal to terminal in the bilayer, the chains would need to tilt to $47.2^{\circ}$ with respect to the sphingosine chain. CERs such as CER NP (C24) have previously been reported to be capable of symmetrically tilting either in their crystalline solid phases over a range of 39$50^{\circ}, 51,52$ as well as when part of a lipid matrix model with a shorter repeat distance, with a symmetric V-shape tilt at an angle of $41^{\circ}$ relative to the membrane normal. ${ }^{53}$ In both situations, the CER chains were symmetrically positioned with respect to the membrane normal. However, it is unlikely that the CERs in our models would have a similar arrangement, due to the fact that the sphingosine group and the acyl chains of the CER NS located in the outer part of the LPP are arranged perpendicular to the headgroup region in the LPP structure. Thus, causing a difference in the chain angle between the two layers would result in a difference in the CER headgroup interfacial area. Thus, if this was to transpire, it would significantly destabilize the LPP structure. Alternatively, the acyl chains maybe linear, if they were either opposing a linoleate group from the CER EOS or opposing another acyl chain and were interdigitated. If two $\mathrm{C} 24$ chains were interdigitated, they would need to occupy a length of 1.7$2.0 \mathrm{~nm}$ at the center of the LPP unit cell to fit. Interdigitation of SC lipids has been previously reported in lipid structures including pure CER systems, ${ }^{51}$ the SPP simple ${ }^{54-56}$ and more complex $^{57}$ models, the LPP structure, ${ }^{22,32}$ and in computa- 

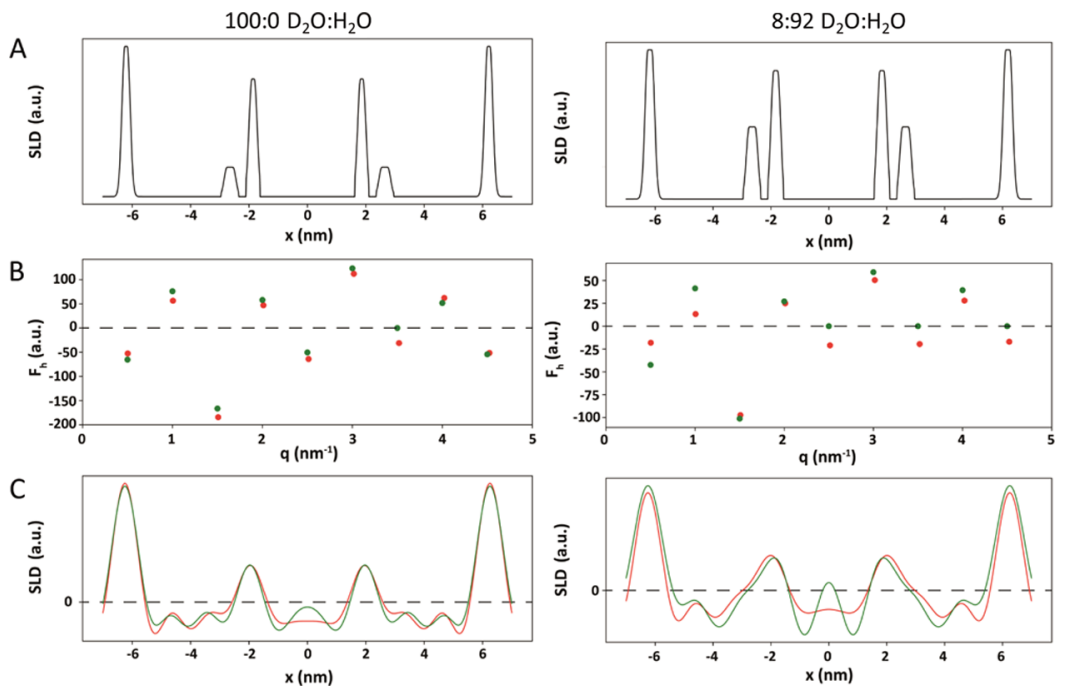

Figure 4. Comparative SLD profiles from experimental (green) and derived (red) data for the simple lipid model when hydrated with 100:0 and 8:92 $\mathrm{D}_{2} \mathrm{O} / \mathrm{H}_{2} \mathrm{O}$. (A) Identified SLD contributions in the LPP unit cell include the inner and outer water boundaries $(6.25,1.9 \mathrm{~nm})$ and the ester group of CER EOS $\left(2.85 \mathrm{~nm}\right.$ ). (B) Calculated $F_{n}$ for each of the Bragg peaks and the calculated $F_{n}$ from the experimental data. (C) Calculated SLD profiles and the SLD profiles calculated from the experimental data.

tional models of the SC lipid matrix. ${ }^{58}$ Out of these options, the partial interdigitation of the acyl chains is the most stable and thus the most likely arrangement of these chains.

Identifying the SLD Contributions. To confirm our understanding of the LPP arrangement and to determine to what extent of the truncation error affected the experimentally obtained SLD profiles, we also calculated the SLD profile using the information on the lipid arrangement from both this experiment and obtained in previous studies. ${ }^{21,22}$ In these calculations, information on the data truncation error was examined, by comparing the calculated with the experimental SLD profiles. In these calculations, the effects of truncation errors were highlighted. By comparing the calculated with the experimental SLD profiles, information can be obtained about the effect of the truncation error. The theoretical SLD profile of the fully protiated simple model hydrated to 100:0 and 8:92 $\mathrm{D}_{2} \mathrm{O} / \mathrm{H}_{2} \mathrm{O}$ was calculated (Figure $4 \mathrm{C}$ ). First, a model of the LPP unit cell was prepared identifying the location of all groups that have a scattering length value (Figure 4A). In the calculated models, these contributions included the water molecules, lipid headgroups, and the ester bond from CER EOS. Due to their almost neutral scattering, the $\mathrm{CH}_{2}$ groups were not included. The water and lipid headgroup contributions were positioned at $6.25 \mathrm{~nm}$ for the outer water region of the LPP and $1.90 \mathrm{~nm}$ for the inner water region. Comparing the SLD intensity between the two hydration states, when the sample was hydrated in $8: 92 \mathrm{D}_{2} \mathrm{O} / \mathrm{H}_{2} \mathrm{O}$, the water scattering is reduced to 0 , leaving the only contribution from the lipids, hence the reduced scattering values. In the 100:0 $\mathrm{D}_{2} \mathrm{O}$ model, approximately an equal number of water molecules were included at each of the headgroup regions. In addition, both hydration conditions included a small broader peak between 2.75 and $2.95 \mathrm{~nm}$ from the center, representing the ester group of the CER EOS. The oxygen in the ester bond contributes to a greater $\operatorname{SLD}$ value at that position $(\mathrm{C}=\mathrm{O} 12.45 \mathrm{fm})$, as opposed to the neutral contribution to the methylene group it otherwise replaces in an acyl chain $\left(\mathrm{CH}_{2}-0.83 \mathrm{fm}\right)$.

From these theoretical SLD contributions, the $F_{n}$ for each order was calculated (Figure 4B; red dots) and compared with the values derived from the experimental data (green dots).
The agreement between the different data sources was generally good. The 7th order of the 100:0 sample and the 5,7 , and 9 th order in the 8:92 sample could not be fitted due to their lack of scattering intensity. First, we wanted to determine the effect of not being able to include the true $F_{n}$ value for these orders in the final SLD profile. The calculated model was able to estimate these values and include these additional $F_{n}$ contributions in its SLD profile (Figure 4C; red curve). Excluding the peaks that could not be quantified in the experimental data, the standard deviation between the $F_{n}$ values, of different sources, for each Bragg peak was $<20 \%$. The following SLD profiles from the predictive calculations (Figure 4C; red curve) matched well with the experimental derived data (green curve), with the only deviation occurring at the center of the unit cell, likely due to truncation error in the experimental derived data. The effect of the Fourier truncation error has been further illustrated in the Supporting Information (Figure S4); by increasing the number of $F_{n}$ values used in the 8:92 model results in a disappearance of the peaks at $-4.5,0$, and $4.5 \mathrm{~nm}$.

Electron Density Profile of the LPP With Temperature. To explore the interplay between the different layers of the LPP, the lengths of the outer and inner layers were monitored at different lateral phases within the simple model. The EDD was calculated from the SAXD profiles (Figure 5A) when the lipids are in the orthorhombic phase $\left(25^{\circ} \mathrm{C}\right)$, hexagonal phase $\left(39^{\circ} \mathrm{C}\right)$, hexagonal with a smaller population of the lipids in the fluid phase $\left(61^{\circ} \mathrm{C}\right)$, and fluid phase with a smaller population of lipids in the hexagonal phase $\left(67^{\circ} \mathrm{C}\right)$, as determined by FTIR. ${ }^{24}$ The black curve of Figure $5 \mathrm{~B}$ shows that at $25{ }^{\circ} \mathrm{C}$, the EDD profile identifies the position of the layer boundaries at similar positions, demonstrated in the SLD profile, with the outer layer located $6.4 \mathrm{~nm}$ from the center of the unit cell and the inner layer at $1.89 \mathrm{~nm}$. The EDD at each of these locations is at a similar intensity to each other, implying a similar electron density at the lipid headgroup regions. In addition, the electron density intensity was greater within the inner layer, when compared to the outer layers. In the outer region, the electron density is particularly low close to the inner headgroup region, where the CHOL is located. ${ }^{22}$ 

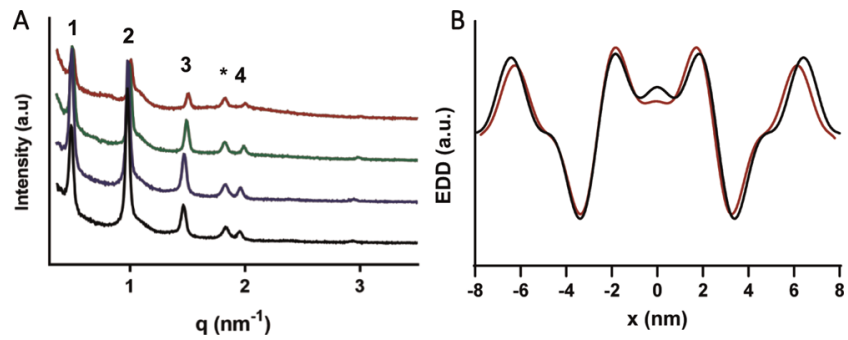

Figure 5. (A) SAXD peaks of the simple system in the orthorhombic $\left(25^{\circ} \mathrm{C}\right.$, black curve), hexagonal $\left(39^{\circ} \mathrm{C}\right.$, blue curve $)$, hexagonal with a smaller population in the fluid $\left(61^{\circ} \mathrm{C}\right.$, green curve), and mixed hexagonal and fluid $\left(67^{\circ} \mathrm{C}\right.$, red curve) phases. The curves have been stacked, for ease of peak observation. (B) Electron density distribution profiles in the orthorhombic phase (black) and in the fluid with the hexagonal phase (red). The position of the highest electron density intensity describes the position of the lipid headgroups and the boundaries of the trilayer.

As the lateral order of the lipids decreased with temperature, the overall length of the LPP unit cell slightly decreases; however, no large change to the length ratios between the individual layers was observed (Table 3). Approaching the

Table 3. Temperature, LPP Length, and the Percent of Length Occupied by Each Layer in the LPP in the Simple Model, When Packed in the Orthorhombic, Hexagonal, Hexagonal with a Small Proportion in the Fluid phase (Hexagonal with Fluid) and When a Greater Proportion of the Lipids are in the Fluid Phase (Fluid with Hexagonal)

\begin{tabular}{clccc}
$\begin{array}{c}\text { temperature } \\
\left({ }^{\circ} \mathrm{C}\right)\end{array}$ & lateral packing & $\begin{array}{c}\text { LPP } \\
\text { length } \\
(\mathrm{nm})\end{array}$ & $\begin{array}{c}\text { outer layer } \\
\text { length } \%)\end{array}$ & $\begin{array}{c}\text { inner layer } \\
\text { length (\%) }\end{array}$ \\
25 & orthorhombic & 12.8 & 35.7 & 28.6 \\
39 & hexagonal & 12.8 & 35.8 & 28.4 \\
61 & $\begin{array}{c}\text { hexagonal with } \\
\text { fluid }\end{array}$ & 12.6 & 35.3 & 29.4 \\
67 & $\begin{array}{c}\text { fluid with } \\
\text { hexagonal }\end{array}$ & 12.3 & 35.7 & 28.6 \\
& & & & \\
\hline
\end{tabular}

fluid phase, the Bragg peaks loose intensity and are broader, which reduces the accuracy of the peak fitting. Therefore, the highest temperature analyzed was $67^{\circ} \mathrm{C}$, at which the lipids were in a mixed fluid and hexagonal phase.

The position of CHOL may also act as an additional factor that promotes the linear arrangement of the central CER NS. CHOL is an essential lipid for proper SC barrier function. $\mathrm{CHOL}$ is required for the formation of the LPP and increases the lipid lateral packing density within the unit cell, ${ }^{59-61}$ a difference in behavior compared to the pure phospholipid systems. ${ }^{62,63} \mathrm{CHOL}$ is a bulky molecule, and according to our results and previous investigations, ${ }^{22}$ it is localized in the outer layers of the LPP close to the inner headgroup. At this location, the electron density is low; therefore, due to its bulky group, $\mathrm{CHOL}$ probably results in a lower electron density. When located here, cholesterol neighbors the sphingosine chains of CER NS and is able to interact via Van der Waal interactions while also capable of hydrogen bonding with the CER headgroup $^{32}$ or the ester group of CER EOS. ${ }^{22}$ In contrast, CHOL avoids neighboring with the linoleic chain of CER EOS in the central layer of the LPP. ${ }^{64}$ These observations have been reproduced with simulations, mimicking the LPP organization $^{43,44}$ and found that the majority of CHOL will selectively neighbor with the CER sphingoid group, due to hydrophobic matching between the chains, thus minimizing the potential energy of this configuration. These predictions have also been confirmed experimentally. ${ }^{65}$ As a result, the combination of neighboring with the CER sphingoid group, while avoiding the linoleic acid of CER EOS, maybe an additional driving force for the observed extended CER NS conformation.

\section{CONCLUSIONS}

In the present study, we have investigated the lipid arrangement of CER NS with the use of SAND. We have demonstrated that within the lipid model LPP arrangement, CER NS adopts an interdigitated linear conformation in the center while also located in the outermost region of the LPP. The advantages of having the inner and outer layers bridged together by the CER NS include reduced permeable boundaries, reduced swelling capabilities, greater lipid packing densities, and reduced packing strain. Two common models were investigated in this study, and although the models had different CER subclass compositions, the CER NS exhibited the same behavior. These results highlight the similarities that both the simple and more complex models have with one another, and how the results observed from one may be applied to the other. The results from this study highlight a new aspect of the lipid arrangement, namely, the lipid configuration, that needs to be considered when assessing the alterations observed between healthy and diseased SC and its impact on the skin barrier function.

\section{ASSOCIATED CONTENT}

\section{Supporting Information}

The Supporting Information is available free of charge at https://pubs.acs.org/doi/10.1021/acs.langmuir.0c01992.

Additional data, explanations, and figures including the model composition, calculation for the SLD absolute scale, SAND rocking plot, relative structure factor of the various diffraction orders, molecular arrangement of CER NS when in a linear extended conformation and the Fourier truncation error contribution (PDF)

\section{AUTHOR INFORMATION}

\section{Corresponding Author}

Joke A. Bouwstra - Division of BioTherapeutics, Leiden Academic Centre for Drug Research, University of Leiden, Leiden 2333 CC, The Netherlands; 이이.org/0000-00027123-6868; Phone: 003171527 4208; Email: bouwstra@ chem.leidenuniv.nl; Fax: 0031715274565

\section{Authors}

Charlotte M. Beddoes - Division of BioTherapeutics, Leiden Academic Centre for Drug Research, University of Leiden, Leiden 2333 CC, The Netherlands; 이이.org/0000-00017449-1031

Gert S. Gooris - Division of BioTherapeutics, Leiden Academic Centre for Drug Research, University of Leiden, Leiden 2333 CC, The Netherlands

Fabrizia Foglia - Chemistry Department, Christopher Ingold Laboratories, University College London, London WC1H OAJ, United Kingdom; 이이이.org/0000-0002-2847-3489

Delaram Ahmadi - Pharmaceutical Science Division, King's College London, London WC2R 2LS, United Kingdom; ○ orcid.org/0000-0002-5186-8956 
David J. Barlow - Pharmaceutical Science Division, King's College London, London WC2R 2LS, United Kingdom

M. Jayne Lawrence - Division of Pharmacy and Optometry, Manchester University, Manchester M13 9PL, United Kingdom

Bruno Demé - Institute Laue-Langevin, Grenoble 38000, France

Complete contact information is available at: https://pubs.acs.org/10.1021/acs.langmuir.0c01992

\section{Notes}

The authors declare no competing financial interest.

\section{ACKNOWLEDGMENTS}

We are grateful to Evonik for their kind donation of CERs. We also thank the personnel at the BM26 Dutch-Belgian beamline at the European Synchrotron Radiation Facility (Grenoble, France) and D16 at the Institut Laue-Langevin (Grenoble, France) for awarding and their assistance during the X-ray and Neutron experiments. ILL raw data DOI: 10.5291/ILLDATA.9-02-906.

\section{REFERENCES}

(1) Hannun, Y. A.; Obeid, L. M. Principles of bioactive lipid signalling: lessons from sphingolipids. Nat. Rev. Mol. Cell Biol. 2008, 9, 139-150.

(2) Wang, X. Lipid signaling. Curr. Opin. Plant Biol. 2004, 7, 329336.

(3) Brasaemle, D. L. Thematic review series: Adipocyte Biology. The perilipin family of structural lipid droplet proteins: stabilization of lipid droplets and control of lipolysis. J. Lipid Res. 2007, 48, 25472559.

(4) Proksch, E.; Brandner, J. M.; Jensen, J. M. The skin: an indispensable barrier. Exp. Dermatol. 2008, 17, 1063-1072.

(5) Boddé, H. E.; Kruithof, M. A. M.; Brussee, J.; Koerten, H. K. Visualisation of normal and enhanced $\mathrm{HgCl} 2$ transport through human skin in vitro. Int. J. Pharm. 1989, 53, 13-24.

(6) Talreja, P. S.; Kasting, G. B.; Kleene, N. K.; Pickens, W. L.; Wang, T. F. Visualization of the lipid barrier and measurement of lipid pathlength in human stratum corneum. AAPS PharmSci 2001, 3, 4856.

(7) Bouwstra, J. A.; Gooris, G. S.; van der Spek, J. A.; Bras, W. Structural Investigations of Human Stratum Corneum by Small-Angle X-Ray Scattering. J. Invest. Dermatol. 1991, 97, 1005-1012.

(8) White, S. H.; Mirejovsky, D.; King, G. I. Structure of Lamellar Lipid Domains and Corneocyte Envelopes of Murine Stratum Corneum. An X-ray Diffraction Study. Biochemistry 1988, 27, $3725-3732$.

(9) t'Kindt, R.; Jorge, L.; Dumont, E.; Couturon, P.; David, F.; Sandra, P.; Sandra, K. Profiling and Characterizing Skin Ceramides Using Reversed-Phase Liquid Chromatography-Quadrupole Timeof-Flight Mass Spectrometry. Anal. Chem. 2012, 84, 403-411.

(10) van Smeden, J.; Bouwstra, J. A. Stratum Corneum Lipids: Their Role for the Skin Barrier Function in Healthy Subjects and Atopic Dermatitis Patients. Curr. Probl. Dermatol. 2016, 49, 8-26.

(11) Wertz, P. W.; Miethke, M. C.; Long, S. A.; Strauss, J. S.; Downing, D. T. The Composition of the Ceramides from Human Stratum Corneum and from Comedones. J. Invest. Dermatol. 1985, 84, 410-412.

(12) Motta, S.; Monti, M.; Sesana, S.; Caputo, R.; Carelli, S.; Ghidoni, R. Ceramide Composition of the Psoriatic Scale. Biochim. Biophys. Acta, Mol. Basis Dis. 1993, 1182, 147-151.

(13) van Smeden, J.; Janssens, M.; Gooris, G. S.; Bouwstra, J. A. The Important Role of Stratum Corneum Lipids for the Cutaneous Barrier Function. Biochim. Biophys. Acta, Mol. Cell Biol. Lipids 2014, 1841, 295-313.
(14) Sahle, F. F.; Gebre-Mariam, T.; Dobner, B.; Wohlrab, J.; Neubert, R. H. H. Skin Diseases Associated with the Depletion of Stratum Corneum Lipids and Stratum Corneum Lipid Substitution Therapy. Skin Pharmacol. Physiol. 2015, 28, 42-55.

(15) Bouwstra, J. A.; Gooris, G. S.; Cheng, K.; Weerheim, A.; Bras, W.; Ponec, M. Phase Behavior of Isolated Skin Lipids. J. Lipid Res. 1996, 37, 999-1011.

(16) Bouwstra, J. A.; Gooris, G. S.; Dubbelaar, F. E. R.; Ponec, M. Phase Behavior of Stratum Corneum Lipid Mixtures Based on Human Ceramides: The Role of Natural and Synthetic Ceramide 1. J. Invest. Dermatol. 2002, 118, 606-617.

(17) de Jager, M. W.; Gooris, G. S.; Ponec, M.; Bouwstra, J. A. Lipid mixtures prepared with well-defined synthetic ceramides closely mimic the unique stratum corneum lipid phase behavior. J. Lipid Res. 2005, 46, 2649-2656.

(18) Bouwstra, J. A.; Gooris, G. S.; Dubbelaar, F. E. R.; Ponec, M. Phase Behavior of Lipid Mixtures Based on Human Ceramides: Coexistence of Crystalline and Liquid Phases. J. Lipid Res. 2001, 42, $1759-1770$.

(19) de Jager, M. W.; Gooris, G. S.; Dolbnya, I. P.; Ponec, M.; Bouwstra, J. A. Modelling the Stratum Corneum Lipid Organisation with Synthetic Lipid Mixtures: the Importance of Synthetic Ceramide Composition. Biochim. Biophys. Acta, Biomembr. 2004, 1664, 132140.

(20) Groen, D.; Gooris, G. S.; Bouwstra, J. A. New Insights into the Stratum Corneum Lipid Organization by X-Ray Diffraction Analysis. Biophys. J. 2009, 97, 2242-2249.

(21) Mojumdar, E. H.; Gooris, G. S.; Barlow, D. J.; Lawrence, M. J.; Deme, B.; Bouwstra, J. A. Skin Lipids: Localization of Ceramide and Fatty Acid in the Unit Cell of the Long Periodicity Phase. Biophys. J. 2015, 108, 2670-2679.

(22) Mojumdar, E. H.; Gooris, G. S.; Groen, D.; Barlow, D. J.; Lawrence, M. J.; Demé, B.; Bouwstra, J. A. Stratum Corneum Lipid Matrix: Location of Acyl Ceramide and Cholesterol in the Unit Cell of the Long Periodicity Phase. Biochim. Biophys. Acta, Biomembr. 2016, 1858, 1926-1934.

(23) Eichner, A.; Sonnenberger, S.; Dobner, B.; Hauß, T.; Schroeter, A.; Neubert, R. H. H. Localization of Methyl-Branched Ceramide [EOS] Species within the Long-Periodicity Phase in Stratum Corneum Lipid Model Membranes: A Neutron Diffraction Study. Biochim. Biophys. Acta, Biomembr. 2016, 1858, 2911-2922.

(24) Beddoes, C. M.; Gooris, G. S.; Bouwstra, J. A. Preferential Arrangement of Lipids in the Long Periodicity Phase of a Stratum Corneum Matrix Model. J. Lipid Res. 2018, 59, 2329-2338.

(25) Wertz, P. W.; Downing, D. T. Epidermal Lipids. In Physiology, Biochemistry and Molecular Biology of the Skin; Goldsmith, L. A., Ed.; Oxford University Press: Oxford, 1991; pp 205-235.

(26) Gooris, G. S.; Kamran, M.; Kros, A.; Moore, D. J.; Bouwstra, J. A. Interactions of Dipalmitoylphosphatidylcholine with CeramideBased Mixtures. Biochim. Biophys. Acta, Biomembr. 2018, 1860, 12721281.

(27) Uche, L. E.; Gooris, G. S.; Beddoes, C. M.; Bouwstra, J. A. New Insight into Phase Behavior and Permeability of Skin Lipid Models Based on Sphingosine and Phytosphingosine Ceramides. Biochim. Biophys. Acta, Biomembr. 2019, 1861, 1317-1328.

(28) Gonthier, J.; Barrett, M. A.; Aguettaz, O.; Baudoin, S.; Bourgeat-Lami, E.; Demé, B.; Grimm, N.; Hauß, T.; Kiefer, K.; Lelièvre-Berna, E.; Perkins, A.; Wallacher, D. BerILL: The ultimate humidity chamber for neutron scattering. J. Neutron Res. 2019, 21, $65-76$.

(29) Richard, D.; Ferrand, M.; Kearley, G. Analysis and Visualisation of Neutron-Scattering Data. J. Neutron Res. 1996, 4, 33-39.

(30) Franks, N. P.; Lieb, W. R. The Structure of Lipid Bilayers and the Effects of General Anaesthetics: An X-ray and Neutron Diffraction Study. J. Mol. Biol. 1979, 133, 469-500.

(31) Harroun, T. A.; Katsaras, J.; Wassall, S. R. Cholesterol hydroxyl group is found to reside in the center of a polyunsaturated lipid membrane. Biochemistry 2006, 45, 1227-1233. 
(32) Mojumdar, E. H.; Groen, D.; Gooris, G. S.; Barlow, D. J.; Lawrence, M. J.; Deme, B.; Bouwstra, J. A. Localization of Cholesterol and Fatty Acid in a Model Lipid Membrane: A Neutron Diffraction Approach. Biophys. J. 2013, 105, 911-918.

(33) Wiener, M. C.; King, G. I.; White, S. H. Structure of a fluid dioleoylphosphatidylcholine bilayer determined by joint refinement of $x$-ray and neutron diffraction data. I. Scaling of neutron data and the distributions of double bonds and water. Biophys. J. 1991, 60, 568576.

(34) Nagle, J. F.; Tristram-Nagle, S. Structure of Lipid Bilayers. Biochim. Biophys. Acta, Biomembr. 2000, 1469, 159-195.

(35) Franks, N. P. Structural Analysis of Hydrated Egg Lecithin and Cholesterol Bilayers I. X-ray Diffraction. J. Mol. Biol. 1976, 100, 345358.

(36) Schröter, A.; Kessner, D.; Kiselev, M. A.; Hauß, T.; Dante, S.; Neubert, R. H. H. Basic Nanostructure of Stratum Corneum Lipid Matrices Based on Ceramides [EOS] and [AP]: A Neutron Diffraction Study. Biophys. J. 2009, 97, 1104-1114.

(37) Schmitt, T.; Gupta, R.; Lange, S.; Sonnenberger, S.; Dobner, B.; Hauß, T.; Rai, B.; Neubert, R. H. H. Impact of the Ceramide Subspecies on the Nanostructure of Stratum Corneum Lipids using Neutron Scattering and Molecular Dynamics Simulations. Part I: Impact of CER[NS]. Chem. Phys. Lipids 2018, 214, 58-68.

(38) Opálka, L.; Kováčik, A.; Maixner, J.; Vávrová, K. Omega-OAcylceramides in Skin Lipid Membranes: Effects of Concentration, Sphingoid Base, and Model Complexity on Microstructure and Permeability. Langmuir 2016, 32, 12894-12904.

(39) Uche, L. E.; Gooris, G. S.; Bouwstra, J. A.; Beddoes, C. M. Barrier Capability of Skin Lipid Models: Effect of Ceramides and Free Fatty Acid Composition. Langmuir 2019, 35, 15376-15388.

(40) Bouwstra, J. A.; Honeywell-Nguyen, P. L.; Gooris, G. S.; Ponec, M. Structure of the skin barrier and its modulation by vesicular formulations. Prog. Lipid Res. 2003, 42, 1-36.

(41) Školová, B.; Hudská, K.; Pullmannová, P.; Kováčik, A.; Palát, K.; Roh, J.; Fleddermann, J.; Estrela-Lopis, I.; Vávrová, K. Different Phase Behavior and Packing of Ceramides with Long (C16) and Very Long (C24) Acyls in Model Membranes: Infrared Spectroscopy Using Deuterated Lipids. J. Phys. Chem. B 2014, 118, 10460-10470.

(42) Engberg, O.; Kováčik, A.; Pullmannová, P.; Juhaščik, M.; Opálka, L.; Huster, D.; Vávrová, K. The Sphingosine and Acyl Chains of Ceramide [NS] Show Very Different Structure and Dynamics Challenging Our Understanding of the Skin Barrier. Angew. Chem., Int. Ed. 2020, 37, No. 999.

(43) Wang, E.; Klauda, J. B. Molecular Structure of the Long Periodicity Phase in the Stratum Corneum. J. Am. Chem. Soc. 2019, 141, 16930-16943.

(44) Lundborg, M.; Narangifard, A.; Wennberg, C. L.; Lindahl, E.; Daneholt, B.; Norlén, L. Human skin barrier structure and function analyzed by cryo-EM and molecular dynamics simulation. J. Struct. Biol. 2018, 203, 149-161.

(45) Corkery, R. W.; Hyde, S. T. On the Swelling of Amphiphiles in Water. Langmuir 1996, 12, 5528-5529.

(46) López-Montero, I.; Rodriguez, N.; Cribier, S.; Pohl, A.; Vélez, M.; Devaux, P. F. Rapid Transbilayer Movement of Ceramides in Phospholipid Vesicles and in Human Erythrocytes. J. Biol. Chem. 2005, 280, 25811-25819.

(47) Vávrová, K.; Kováčik, A.; Opálka, L. Ceramides in the Skin Barrier. Eur. Pharm. J. 2017, 64, 28-35.

(48) Paz Ramos, A.; Gooris, G.; Bouwstra, J. A.; Lafleur, M. Evidence of Hydrocarbon Nanodrops in Highly Ordered Stratum Corneum Model Membranes. J. Lipid Res. 2018, 59, 137-143.

(49) Mojumdar, E. H.; Helder, R. W. J.; Gooris, G. S.; Bouwstra, J. A. Monounsaturated Fatty Acids Reduce the Barrier of Stratum Corneum Lipid Membranes by Enhancing the Formation of a Hexagonal Lateral Packing. Langmuir 2014, 30, 6534-6543.

(50) Školová, B.; Kováčik, A.; Tesař, O.; Opálka, L.; Vávrová, K. Phytosphingosine, Sphingosine and Dihydrosphingosine Ceramides in Model Skin Lipid Membranes: Permeability and Biophysics. Biochim. Biophys. Acta, Biomembr. 2017, 1859, 824-834.
(51) Dahlén, B.; Pascher, I. Molecular Arrangements in Sphingolipids. Crystal structure of N-tetracosanoylphytosphingosine. Acta Crystallogr., Sect. B: Struct. Sci. 1972, 28, 2396-2404.

(52) Wartewig, S.; Neubert, R. H. H. Properties of Ceramides and Their Impact on the Stratum Corneum Structure: A Review. Skin Pharmacol. Physiol. 2007, 20, 220-229.

(53) Schroeter, A.; Stahlberg, S.; Skolová, B.; Sonnenberger, S.; Eichner, A.; Huster, D.; Vávrová, K.; Hauß, T.; Dobner, B.; Neubert, R. H. H.; Vogel, A. Phase Separation in Ceramide[NP] Containing Lipid Model Membranes: Neutron Siffraction and Solid-State NMR. Soft Matter 2017, 13, 2107-2119.

(54) Schroeter, A.; Kiselev, M. A.; Hauß, T.; Dante, S.; Neubert, R. H. H. Evidence of Free Fatty Acid Interdigitation in Stratum Corneum Model Membranes Based on Ceramide [AP] by Deuterium Labelling. Biochim. Biophys. Acta, Biomembr. 2009, 1788, 2194-2203.

(55) Schmitt, T.; Neubert, R. H. H. State of the Art in Stratum Corneum Research: The Biophysical Properties of Ceramides. Chem. Phys. Lipids 2018, 216, 91-103.

(56) Schmitt, T.; Lange, S.; Dobner, B.; Sonnenberger, S.; Hauß, T.; Neubert, R. H. H. Investigation of a CER $[\mathrm{NP}]$ - and [AP]-Based Stratum Corneum Modeling Membrane System: Using Specifically Deuterated CER Together with a Neutron Diffraction Approach. Langmuir 2017, 34, 1742-1749.

(57) Groen, D.; Gooris, G. S.; Barlow, D. J.; Lawrence, M. J.; van Mechelen, J. B.; Demé, B.; Bouwstra, J. A. Disposition of Ceramide in Model Lipid Membranes Determined by Neutron Diffraction. Biophys. J. 2011, 100, 1481-1489.

(58) Podewitz, M.; Wang, Y.; Gkeka, P.; von Grafenstein, S.; Liedl, K. R.; Cournia, Z. Phase Diagram of a Stratum Corneum Lipid Mixture. J. Phys. Chem. B 2018, 122, 10505-10521.

(59) Chen, H.; Mendelsohn, R.; Rerek, M. E.; Moore, D. J. Effect of cholesterol on miscibility and phase behavior in binary mixtures with synthetic ceramide 2 and octadecanoic acid. Infrared studies. Biochim. Biophys. Acta, Biomembr. 2001, 1512, 345-56.

(60) Mojumdar, E. H.; Gooris, G. S.; Bouwstra, J. A. Phase behavior of skin lipid mixtures: the effect of cholesterol on lipid organization. Soft Matter 2015, 11, 4326-4336.

(61) Das, C.; Noro, M. G.; Olmsted, P. D. Simulation Studies of Stratum Corneum Lipid Mixtures. Biophys. J. 2009, 97, 1941-1951.

(62) Fenske, D. B.; Thewalt, J. L.; Bloom, M.; Kitson, N. Models of stratum corneum intercellular membranes: $2 \mathrm{H}$ NMR of macroscopically oriented multilayers. Biophys. J. 1994, 67, 1562-1573.

(63) Kitson, N.; Thewalt, J.; Lafleur, M.; Bloom, M. A model membrane approach to the epidermal permeability barrier. Biochemistry 1994, 33, 6707-15.

(64) McIntosh, T. J. Organization of Skin Stratum Corneum Extracellular Lamellae: Diffraction Evidence for Asymmetric Distribution of Cholesterol. Biophys. J. 2003, 85, 1675-1681.

(65) Ouimet, J.; Lafleur, M. Hydrophobic Match between Cholesterol and Saturated Fatty Acid Is Required for the Formation of Lamellar Liquid Ordered Phases. Langmuir 2004, 20, 7474-7481. 